\title{
Archéopages
}

Archéopages

Archéologie et société

$44 \mid 2017$

Terrains vagues

\section{Fabrication et fonction des outils de type montmorencien. Nouveau regard à partir des découvertes récentes sur les habitats mésolithiques}

Sylvain Griselin

\section{CpenEdition}

Journals

Édition électronique

URL : https://journals.openedition.org/archeopages/1599

DOI : 10.4000/archeopages. 1599

ISSN : 2269-9872

Éditeur

INRAP - Institut national de recherches archéologiques préventives

Édition imprimée

Date de publication : 1 juin 2017

Pagination : $92-93$

ISSN : $1622-8545$

\section{Référence électronique}

Sylvain Griselin, «Fabrication et fonction des outils de type montmorencien. Nouveau regard à partir des découvertes récentes sur les habitats mésolithiques », Archéopages [En ligne], 44 | 2017, mis en ligne le 01 juillet 2019, consulté le 02 juin 2021. URL : http://journals.openedition.org/archeopages/ 1599 ; DOI : https://doi.org/10.4000/archeopages.1599 


\section{Sylvain Griselin \\ Inrap, UMR 7041, «Arscan » \\ $1^{\text {ER }}$ DÉCEMBRE 2015 \\ Université Paris I \\ Panthéon-Sorbonne \\ Directeur de thèse \\ Boris Valentin \\ Professeur, université Paris I \\ Panthéon-Sorbonne \\ Membres du jury \\ Fabrication et fonction des outils de type montmorencien. Nouveau regard à partir des découvertes récentes sur les habitats mésolithiques.}

Président

Jacques Pelegrin

Directeur de recherche, CNRS

Rapporteur

Grégor Marchand

Directeur de recherche, CNRS

Examinateurs

Pierre Bodu

Chargé de recherche, CNRS

Annelou van Gijn

Professeure, université de Leyde,

Pays-Bas

Caroline Hamon

Chargée de recherche, CNRS

Frédéric Séara

Conservateur régional

de l'archéologie de la région

Grand Est

Consultable

sous format papier à l'université

de Paris I Panthéon-Sorbonne.

Publication en cours.
Les industries macrolithiques en grès ont, depuis les premières découvertes à la fin du $\mathrm{XIX}^{\mathrm{e}}$ siècle, interpellé les chercheurs, en particulier sur la question de leur attribution chronoculturelle. Il faut attendre la fin des années 1970 pour qu'une importante synthèse sur ces industries soit réalisée par Jacques Tarrête (en 1977), les rapportant à un faciès singulier au sein des industries post-glaciaire : le Montmorencien. Cette synthèse met en évidence l'importance des productions de pièces macrolithiques allongées en grès-quartzite dans l'espace francilien, le long des affleurements de grès stampien, sans pour autant permettre de préciser leur place chronologique, même si une attribution au Mésolithique est évoquée. Les problématiques liées à la présence d'outils en grès-quartzite au sein d'assemblages mésolithiques ressurgissent dans les années 1990 grâce à de nouvelles découvertes sur des habitats, notamment ceux fouillés dans le cadre de l'archéologie préventive. On trouve sur ces habitats des outils macrolithiques allongés en grès-quartzite qui induisent un rapport particulier entre le Mésolithique et le Montmorencien. C'est donc pour définir la place de ces outils au sein de la culture matérielle du Mésolithique et leur rapport avec le Montmorencien que nous avons repris l'étude d'outils trouvés en contexte d'habitat et en particulier sur des sites fouillés ces 10 dernières années par l'Inrap comme celui du 62, rue Henry-Farman à Paris (responsable d'opération : Bénédicte Souffi, Inrap). Nous nous sommes interrogés sur les savoir-faire techniques investis pour la production des pièces, sur l'objectif et l'utilisation de cette production.
Notre analyse montre que les outils en grès utilisés dans les habitats mésolithiques correspondent à des macrolithes allongés, bien typés au sein des corpus, que nous avons appelés outils de type montmorencien. Ces outils forment une catégorie relativement standardisée par ses dimensions et par l'attention portée au cours du façonnage puis lors de leur entretien. Il s'agit d'objets qui mesurent une dizaine de centimètres de longueur pour une largeur et une épaisseur de deux à trois centimètres. Ils présentent une section prismatique liée à l'existence de trois à cinq faces dont l'une au moins est plane, dépourvue de retouche. Le façonnage vise à la mise en place de deux ou trois arêtes latérales plutôt régulières sur toute la longueur des pièces. Les réfections observées ont pour rôle l'entretien de ces arêtes alors que les extrémités ne jouent qu'un rôle fonctionnel secondaire. De plus, l'importance des réfections observées le long des arêtes indique que les outils sont conçus pour être utilisés pendant une durée relativement longue. L'analyse tracéologique réalisée par Caroline Hamon (CNRS) montre que les arêtes sont employées sur une matière minérale dure et des tests expérimentaux nous orientent vers une utilisation comme retouchoirs pour la fracturation des lamelles par la technique du microburin. On peut présenter cette hypothèse comme la plus crédible, même si des précisions quant aux gestes employés devront être apportées en poursuivant les expérimentations. Dans les habitats mésolithiques, on trouve souvent ces outils au sein d'assemblages microlithiques dominés par les pointes à base retouchée et les segments du Beuronien nord-occidental, datés entre 8700 et 7500 avant notre ère. Ils sont principalement présents sur les sites franciliens et, plus rarement, jusqu'aux marges du Bassin parisien. Ainsi, ces outils caractérisent un «phénomène technique » d'ampleur régionale, voire suprarégionale au cours du premier Mésolithique.

L'étude engagée pour une révision de séries montmorenciennes montre bien qu'une partie importante des pièces sont similaires, morphologiquement et technologiquement, à celles des habitats mésolithiques. Elles sont toutefois inachevées dans ces séries et ne présentent pas de traces macroscopiques d'utilisation identiques à celles trouvées sur les habitats mais d'autres, probablement consécutives à leur emploi au cours de procédés d'extraction, ce qui cadre bien avec la vocation « productrice » de ces gisements. En effet, ces sites que nous qualifions de " producteurs », ou montmorenciens stricto sensu, sont localisés sur ou à proximité des gîtes de grèsquartzite où le matériau est extrait pour ensuite $y$ façonner des outils, emportés ultérieurement sur les habitats mésolithiques. On observe aussi de manière marginale la présence d'ébauches apportées dans certains habitats mésolithiques. Ces pièces 
pourraient révéler un comportement singulier de mise en réserve de quelques supports, utilisés au gré des besoins. Les études lithologiques à venir devront définir les modalités liées à la diffusion de ces supports et des outils à travers les territoires.

Par ailleurs, nous avons pu mettre en évidence sur la plupart des sites montmorenciens stricto sensu la présence de différents types d'ébauches de haches néolithiques, nous amenant à requalifier le « Montmorencien ». Celui-ci ne peut plus, selon nous, désigner un faciès chronologique spécifique, puisque l'on trouve au sein de ses séries des pro- ductions tant néolithiques que mésolithiques. Il n'a donc plus qu'une valeur historiographique. Pour la caractérisation des industries mésolithiques, nous proposons de restreindre son usage comme adjectif pour désigner un type d'outil marqueur d'un comportement singulier au cours du premier Mésolithique. L'utilisation de ces outils comme retouchoirs pour une activité singulière, possiblement liée à la segmentation des lamelles par la technique du microburin, est révélatrice d'une "économie des matières premières » du fait de l'emploi spécifique du grès-quartzite pour leur confection.

MARS 2013
Université Paris I
Panthéon-Sorbonne
Directeur de thèse
Jean-Paul Demoule
Professeur émérite,
Université Paris I Panthéon-Sorbonne
Membres du jury
Président
Patrice Brun
Professeur, université Paris I
Panthéon-Sorbonne
Rapporteurs
Bruno Boulestin
Chercheur associé,
Université de Bordeaux I
Stéphane Verger
Directeur d'études, École Pratique
des Hautes Études
Examinatrice
Isabelle Le Goff
Ingénieure chargée d'études, Inrap
Consultable
sous format papier à I'université
de Paris I Panthéon-Sorbonne.
Mate

\section{Lola Bonnabel}

Inrap, UMR 8215 « Trajectoires »

\section{Approche anthropologique de la société Aisne-Marne à partir de ses pratiques mortuaires dans le cadre de l'archéologie préventive (Champagne-Ardenne, $\mathrm{VI}^{\mathrm{e}}$-III ${ }^{\mathrm{e}}$ siècle avant notre ère).}

Le travail de thèse s'est appuyé sur un programme collectif regroupant des chercheurs (Inrap, services régionaux de l'Archéologie) ayant participé aux différentes phases de l'archéologie préventive, de la prescription de la fouille à la publication des études. Le corpus ainsi constitué est de 599 défunts appartenant pour la plupart à La Tène A et B, mais également pour une partie d'entre eux au Hallstatt final et à La Tène $\mathrm{C}$. Les restes de 552 d'entre eux (dont cinq préalablement crémés) proviennent de tombes appartenant à 28 occupations funéraires pouvant regrouper de 1 à 83 défunts, tandis que les cadavres de 47 de ces décédés ont été placés dans une ancienne structure de conservation du grain. La culture archéologique principalement illustrée est celle de l'Aisne-Marne qui se développe entre ces deux rivières et a été définie par le travail fondateur de Jean-Paul Demoule, mais notre corpus dépasse les frontières de cette culture vers le sud et nous permet d'en souligner les spécificités.

La thèse s'est concentrée sur les données issues de l'archéologie préventive. Les deux raisons principales étaient que les travaux plus anciens avaient déjà été parfaitement exploités par J.-P. Demoule et que j'avais l'occasion d'étudier un ensemble méthodologiquement cohérent. Ce choix devait permettre d'apporter un point de vue nouveau. Il métait important de voir comment extraire des hypothèses sociales de ces données soigneusement classées, étudiées par chacune et chacun d'entre nous. C'est-à-dire que si je désirais trouver une façon pertinente de présenter les nombreuses informations que nous avions extraites du terrain et des études informant sur les us et coutumes des populations de l'âge du Fer en matière mortuaire (une sorte de travail ethnographique), j'aspirais également à pouvoir en déduire des propositions interprétatives sur l'organisation des sociétés et leur idéologie.

Cette thèse est construite en quatre parties. La première est un plan général, elle s'intéresse aux données du corpus dans leur contexte, en particulier géographique et archéologique. Elle est l'occasion de montrer qu'il existe un « territoire » de l'AisneMarne se distinguant d'une « culture » Aisne-Marne plus étendue. Les opérations de diagnostic systématique montrent les frontières est, nord et sud de ce territoire.

La deuxième partie est un plan d'ensemble où sont examinés les lieux où les morts sont rassemblés, ou parfois isolés. Les défunts de la période Hallstatt sont inhumés en nécropoles, plusieurs fois installées sur des nécropoles antérieures marquant le paysage de leurs imposants monuments funéraires. Au fil de La Tène ancienne, des nécropoles aux tombes plus nombreuses sont implantées, elles s'organisent suivant des règles communes d'alignements, d'enfilades, de vides préservés, qui perdurent tout au long de leur constitution. Ces dispositions normées et les superpositions semblent indiquer que chaque individu a une place définie par sa position au sein d'un groupe. Un discours plus ou moins élitaire ou égalitaire semble exprimé en fonction de ces ensembles, les nécropoles élitaires se rencontrant plus particulièrement en frontière nord et sudest du domaine Aisne-Marne. À la fin de La Tène ancienne, durant La Tène moyenne, les nécropoles sont désaffectées, les défunts sont inhumés isolés ou en petits groupes de tombes. Une part des défunts sont inhumés en structure de stockage réutilisée, en proportion plus importante lors des dernières phases de La Tène ancienne. La partie sud de la région Champagne-Ardenne, qui n'appartient pas à ce que nous avons défini comme le territoire AisneMarne, se distingue du reste avec une plus grande importance de ce type d'inhumation et une rareté des nécropoles qui regroupent moins de défunts.

La troisième partie est un plan rapproché sur la structure où les corps de ces défunts sont placés, qu'il s'agisse d'une tombe ou d'un silo. C'est l'occasion d'examiner ce que nous pouvons reconstituer de 